\title{
Assessment of the Adequacy of Curriculum Content of Mechanical Technology Programme Implementation in Technical Colleges of Kaduna and Niger States, Nigeria
}

\author{
Ishaya Ibrahim Bature, Umar Abubakar
}

\begin{abstract}
The purpose of this study was to assess the adequacy of the curriculum content of mechanical technology programme implementation in technical colleges of Kaduna and Niger states, Nigeria. To conduct the study a research question was formulated. Descriptive survey design was adopted for the study. The population was 61 comprising 49 mechanical technology teachers and 12 heads of department. There was no sampling as the population size was manageable. A structured questionnaire containing 67 items was used for data collection. The instrument was face validated by three experts. Mean was used to answer the one research question. One of the major findings of the study revealed that the curriculum content of mechanical technology was inadequate. The study recommended among others that the curriculum content of mechanical technology be reviewed in order to meet up with the present technological challenges affecting all facets of human endeavours globally.
\end{abstract}

Index Terms - Assess, Adequacy, Curriculum Content, Mechanical Technology Technical Colleges, Kaduna and Niger States, Nigeria.

\section{INTRODUCTION}

Highlight In order to ascertain the adequacy of curriculum content of any training programme being implemented in a technical college, it has to be assessed. That is, one has to make judgement about the quality of the programme. By adequacy of curriculum content here we mean those things which the students have to learnt at a stipulated time in the course of their studies, should have an indepth coverage of subject matter relevant to their teaching-learning needs.

If the curriculum content is inadequate the learners will become half-baked at the end of the training programme (Bature, 2011). Hence, the students would not be able to acquire the necessary skills that could warrant them employment opportunity or for further education.

Undoubtedly, one of the objectives of implementing mechanical technology in the technical colleges according to Mupinga in Bature (2011) is to develop in the learner the

ISHAYA IBRAHIM BATURE (PhD), Department Of Education (Technical), College Of Science And Technology, Kaduna Polytechnic, P.M.B. 2021 , Kaduna - Nigeria

UMAR ABUBAKAR (PhD), Department Of Education (Technical), College Of Science And Technology, Kaduna Polytechnic, P.M.B. 2021 , Kaduna - Nigeria ability to do something to earn a living. That is, the students should be able to manufacture those things that are useful in the society. Also, during their course of studies the students were expected to be able to produce among other things simple engineering components using various tools, machines and equipment. However, as observed in 2016 by the researchers most of the graduates of mechanical technology from technical colleges of Kaduna and Niger states were seeing roaming about the streets of towns and villages without being employed. These graduands were supposed to be self-employed or hired. But the reverse is the case in Kaduna and Niger states.

It would seem there are a number of factors that might have contributed to the students lack of been self-employed or hired. Bature (2011) observed that most of the technical colleges are faced with the following problems, especially during implementation namely:

1. Insufficient funds

2. Insufficient practical materials

3. Insufficient machines, equipment and tools

4. Insufficient qualified and experienced technical teachers.

Consequently, if the problems mentioned above are not solved there is the tendency that the students are bound to become half-baked trained graduants.

Furthermore, apart from the problem of inadequate training facilities as observed by the researchers (2017), one other big challenge could be attributed to the curriculum content of the mechanical technology is whether it has wide and indepth coverage which meets the needs of the students. Hence the need of this present study is to assess the adequacy of the curriculum content of mechanical technology programme implementation in technical colleges of Kaduna and Niger states, Nigeria.

\section{Purpose of the Study}

The purpose of this study was to assess the adequacy of curriculum content of mechanical technology programme implementation in technical colleges of Kaduna and Niger states, Nigeria.

Research Question

How adequate are the curriculum contents of mechanical technology programme implementation in technical colleges of Kaduna and Niger states, Nigeria in meeting the job requirements of the industries where the graduates will work?

Hypothesis

The following null hypothesis was formulated and tested 


\section{Assessment of the Adequacy of Curriculum Content of Mechanical Technology Programme Implementation in Technical Colleges of Kaduna and Niger States, Nigeria}

at the 0.05 level of significance in the study:

Ho: There is no significant difference between the mean response of teachers and heads of department on the adequacy of the curriculum content of mechanical technology.

\section{METHODOLOGY}

An 67-item questionnaire was developed for this study by the researchers through an intensive literature review. This study adopted a descriptive survey research design. The study was conducted in Kaduna and Niger states, Nigeria. The population for this study comprises of 49 teachers and 12 heads of department. Thus the entire population was 61 . The whole population was used since the size of the population was manageable. Therefore there was no sampling. Three experts from the Department of Industrial Technical Education University of Nigeria Nsukka validated the instrument. The analysis of data for this study was done using mean. Thus any item with a mean value or greater than 3.00 were accepted otherwise rejected.

\section{RESULTS}

The results of the research question are presented in the table below.

\section{Research Question}

How adequate are curriculum contents of mechanical technology programme implementation in Technical Colleges of Kaduna and Niger states, Nigeria in meeting the job requirement of the industries where the graduates will work?

Answers to this research question are presented in table 1 below.

Table 1 Mean and Standard Deviation responses of teachers and Heads of Department on the Adequacy of Curriculum Content of Mechanical Technology Programme.

$\begin{array}{lllrl}\text { S/No } & \text { Items } & \text { N=61 } & \text { S.D } & \text { Decision } \\ & \text { Adequacy of Curriculum Contents of Mechanical Technology } & & \end{array}$

Adequacy of Curriculum Contents of Mechanical Technology

$\overline{\boldsymbol{X}}$

\section{Bench Work}

Principles of bench clamping devices such as bench vice, leg vice etc

Methods of controlling clamping pressure

The identification of description of the types of grinders

Characteristics of good grinding stone

Fitting work

Bench operations of shaping metals to size, etc,

Common fitting tools their cutting actions and uses.

The basic action of cutting metals by sawing, shearing

The types and parts of tools like, chisel, hacksaw, scissors, etc

Cutting principles of shearing metals to size

The principle of filing and filing actions.

The principles of shaping metals by chiseling

Highlight on the importance, types and uses of hammers in metal fitting

\section{Fabrication /Welding}

The equipment used in gas welding

The equipment used in metals arc welding

The safety precautions to be observed and applied to welding situations

The procedures to be employed in welding surfaces using oxy-acetylene torch

The procedures to be implored in welding surfaces using electric arc.

\section{Forging}

The main features of the blacksmith's forge

The working principles of the black smith forge.

The uses of common forging tools such as anvil, swage blocks, etc

The procedures to be implored in carry out forging operations such as upsetting, drawing down etc.

\section{Foundry}

The factory safety act for foundry practices

The use of productive equipment in the foundry shop.

The identification of foundry tools and devices

The principles, types and techniques of foundry works.

The uses of pattern in foundry works

The characteristics of patterns

The working principles of common pattern making tools

Lathe Machine Work

The identification of the types of lathe machines

$\begin{array}{lll}3.21 & .97 & \text { Fairly adequate } \\ 2.96 & 1.10 & \text { Not adequate } \\ 2.64 & .91 & \text { Not adequate } \\ 2.89 & .99 & \text { Not adequate }\end{array}$

2.71

1.05

$2.96 \quad .88$

Not adequate

$2.61 \quad .88$

$2.57 \quad .88$

$2.68 \quad 1.06$

$2.93 \quad .84$

$3.04 \quad 1.20$

$3.00 \quad .90$

Not adequate

Not adequate

Not adequate

Not adequate

Not adequate

Fairly adequate

Fairly adequate

Not adequate

Not adequate

Not adequate

Not adequate

Fairly adequate Fairly adequate Fairly adequate Not adequate

$\begin{array}{lll}2.71 & .90 & \text { Not adequate } \\ 3.14 & .71 & \text { Fairy adequate } \\ 3.21 & .79 & \text { Fairy adequate } \\ 3.07 & .98 & \text { Fairy adequate } \\ 3.14 & .93 & \text { Fairy adequate } \\ 3.14 & .76 & \text { Fairy adequate } \\ 3.14 & .76 & \text { Fairy adequate } \\ & & \text { Fairy adequate }\end{array}$




\begin{tabular}{|c|c|c|c|c|}
\hline 30 & The working principles of lathe machine & 2.57 & .96 & Not adequate \\
\hline 31 & $\begin{array}{l}\text { The function and constructional details of the component parts of each type of } \\
\text { lathe machines and its accessories. }\end{array}$ & 2.39 & 1.03 & Not adequate \\
\hline 32 & The problems associated with the machining of plastics & 2.64 & .87 & Not adequate \\
\hline 33 & The various types of working/holding equipment used on the centre lathe & 3.00 & .86 & Fairy adequate \\
\hline 34 & $\begin{array}{l}\text { Explanation on how to determine a work plan from turning job } \\
\text { Milling Machine Work }\end{array}$ & 2.96 & 1.00 & Not adequate \\
\hline 35 & The Identification of the types of Milling Machines & 2.93 & 1.15 & Not adequate \\
\hline 36 & The working principles of a milling machine & 2.61 & .88 & Not adequate \\
\hline 37 & The basic maintenance activities on a milling machine & 2.61 & .96 & Not adequate \\
\hline 38 & $\begin{array}{l}\text { The functions and constructional details of component parts of each milling } \\
\text { machine and its accessories } \\
\text { Shaping Machine Work }\end{array}$ & 2.39 & .99 & Not adequate \\
\hline 39 & The identification of the types of shaping machine and set up tools & 2.64 & 1.10 & Not adequate \\
\hline 40 & How to set and operate the shaper to produce various components & 2.54 & .84 & Not adequate \\
\hline 41 & How to maintain the shaping machine & 2.50 & .75 & Not adequate \\
\hline 42 & $\begin{array}{l}\text { The functions and constructional details of the main components of the shaper } \\
\text { machine } \\
\text { Design }\end{array}$ & 2.46 & .84 & Not adequate \\
\hline 43 & The principle of basic design & 2.64 & .95 & Not adequate \\
\hline 44 & The identification of materials used in design & 2.96 & .84 & Not adequate \\
\hline 45 & $\begin{array}{l}\text { The identification and description of tools used in design } \\
\text { Finishing }\end{array}$ & 2.96 & .79 & Not adequate \\
\hline 46 & The procedures of finishing up a stock with a portable filing machine & 3.03 & .84 & Fairly adequate \\
\hline 47 & The procedures of finishing up a stock with an abrasive bell grinder. & 2.86 & .80 & Not adequate \\
\hline 48 & $\begin{array}{l}\text { The procedures of finishing up a stock with a buffing machine } \\
\text { Soldering/Brazing }\end{array}$ & 2.93 & .86 & Not adequate \\
\hline 49 & The composition of soft solders & 2.96 & .96 & Not adequate \\
\hline 50 & The principles of soldering & 2.86 & .76 & Not adequate \\
\hline 51 & The identification of factors that determine if a job is to be soldered. & 2.82 & .77 & Not adequate \\
\hline 52 & The description of soldering equipment and their functions & 2.82 & .82 & Not adequate \\
\hline 53 & The description of the purpose of fluxes & 3.00 & .94 & Fairly adequate \\
\hline 54 & The description of how to test soldered joints for rigidity and leakage & 3.11 & .77 & Fairly adequate \\
\hline 55 & $\begin{array}{l}\text { How to differentiate between the melting points of solder and metal } \\
\text { Hammering Metal Work }\end{array}$ & 2.93 & .77 & Not adequate \\
\hline 56 & The procedures for annealing & 3.18 & .72 & Fairly adequate \\
\hline 57 & The procedures for hollowing & 2.93 & .86 & Not adequate \\
\hline 58 & The procedures for sinking & 3.00 & .77 & Fairly adequate \\
\hline 59 & The procedures for rising & 3.07 & .72 & Fairly adequate \\
\hline 60 & $\begin{array}{l}\text { The identification of equipment and tools used in foundry } \\
\text { Measuring/ Marking out }\end{array}$ & 3.01 & .74 & Fairly adequate \\
\hline 61 & $\begin{array}{l}\text { The essential features and use of measuring tools such as micrometer screw } \\
\text { guage, etc }\end{array}$ & 3.14 & .76 & Fairly adequate \\
\hline 62 & How to care and maintain measuring and making out tools & 3.25 & .80 & Fairly adequate \\
\hline 63 & $\begin{array}{l}\text { The functions and application of making tools such as rule, dividers, caliper etc } \\
\text { Riveting }\end{array}$ & 2.93 & .86 & Not adequate \\
\hline 64 & The procedures of cold riveting a work piece on the work bench & 3.14 & .93 & Not adequate \\
\hline 65 & The procedures of hot riveting a work piece with a hand riveting machine & 3.04 & .43 & Not adequate \\
\hline 66 & The procedures of riveting a work piece using a powered riveting machine & 3.18 & .72 & Not adequate \\
\hline 67 & The procedures of riveting a work piece using a portable pneumatic press. & 3.07 & 1.05 & Not adequate \\
\hline
\end{tabular}

The result in table 1 shows that the respondents rated the following items in the mechanical technology curriculum content main themes as fairly adequate $1,11,18,18,19,20,23,24,25,26,27,28,29,32,46,53,54,56,57,58,59,60,61,62,64,65,66$ and 67 . However, items, 2, 3, 4,5, 6,7,8,9,10,13,14,15,16, 21, 22, 30, 31, 32, 34, 36,37,38,39,40,41,42,43,44,45,47,48,49,50, 51, 52, 55,57 , and 65 were rated as being inadequate.

\section{Hypothesis}

There is no significant difference between the mean response of teachers and heads of department on the adequacy of the curriculum content of mechanical technology.

Data for $\mathrm{H}_{0}$ is presented in table 2. 
Table 2 T-test of difference between the mean responses of Teachers and Heads of Department regarding the Adequacy of Curriculum Content of Mechanical Technology programme in Technical Colleges

\begin{tabular}{|c|c|c|c|c|c|c|c|c|c|c|}
\hline $\begin{array}{l}\text { Dependent } \\
\text { variables }\end{array}$ & & Status & $\mathbf{N}$ & Mean & $\begin{array}{l}\text { Std } \\
\text { Deviation }\end{array}$ & $\begin{array}{l}\text { Std. } \\
\text { Error } \\
\text { Mean } \\
\end{array}$ & Df. & t-Cal & $\begin{array}{r}\text { t- } \\
\text { Crit }\end{array}$ & $\begin{array}{l}\text { Decis } \\
\text { ions }\end{array}$ \\
\hline 1 Work on & Item 1 & HOD & 12 & 3.5000 & 1.259099 & .64550 & 59 & 0.746 & 1.960 & NS \\
\hline \multirow[t]{9}{*}{ Bench } & & Teachers & 49 & 3.1667 & .91683 & .18715 & & & & \\
\hline & Item 2 & HOD & 12 & 2.7500 & .99 .743 & .47871 & 59 & 0.211 & 1.960 & NS \\
\hline & & Teachers & 49 & 3.0000 & 1.4208 & .23313 & & & & \\
\hline & Item 3 & HOD & 12 & 3.0000 & .81650 & .40825 & 59 & 0.817 & 1.960 & NS \\
\hline & & Teachers & 49 & 2.5833 & .92861 & .18955 & & & & \\
\hline & Item 4 & HOD & 12 & 3.5000 & 1.0000 & .50000 & 59 & .494 & 1.960 & NS \\
\hline & & Teachers & 49 & 2.7917 & 977098 & .19945 & & & & \\
\hline & Overall & HOD & 12 & 3.1875 & .87500 & .43750 & 59 & 0.817 & 1.960 & NS \\
\hline & & Teachers & 49 & 2.8854 & .84692 & .17288 & & & & \\
\hline $2 \quad$ Fitting & Item 5 & HOD & 12 & 3.2500 & 1.25831 & .62916 & 59 & 2.093 & 1.960 & S \\
\hline \multirow[t]{15}{*}{ Work } & & Teachers & 49 & 2.6250 & 1.01350 & .20688 & & & & \\
\hline & Item 6 & HOD & 12 & 3.2500 & 1.50000 & .75000 & 59 & 0.300 & 1.960 & NS \\
\hline & & Teachers & 49 & 2.9167 & .77553 & .15830 & & & & \\
\hline & Item 7 & HOD & 12 & 2.50000 & 1.29099 & .64550 & 59 & -0.081 & 1.960 & NS \\
\hline & & Teachers & 49 & 2.6250 & .82423 & .16824 & & & & \\
\hline & Item 8 & HOD & 12 & 2.2500 & .95743 & .47871 & 59 & -0.351 & 1.960 & NS \\
\hline & & Teachers & 49 & 2.6250 & .87539 & .17869 & & & & \\
\hline & Item 9 & HOD & 12 & 2.2500 & .95743 & .47871 & 59 & -0.471 & 1.960 & NS \\
\hline & & Teachers & 49 & 2.7500 & 1.07239 & .21911 & & & & \\
\hline & Item 10 & HOD & 12 & 2.5000 & 1.29099 & .64550 & 59 & 0.408 & 1.960 & NS \\
\hline & & Teachers & 49 & 3.0000 & .88465 & .1805 & & & & \\
\hline & Item 11 & HOD & 12 & .81650 & .81650 & .40825 & 59 & 1.288 & 1.960 & NS \\
\hline & & Teachers & 49 & 1.1919 & 1.29100 & .24311 & & & & \\
\hline & Item 12 & HOD & 12 & 3.2500 & .2500 & .2500 & 59 & 0.432 & 1.960 & NS \\
\hline & & Teachers & 49 & 2.9583 & .95458 & .19485 & & & & \\
\hline 3 & Item 13 & HOD & 12 & 3.2500 & .50000 & 25000 & 59 & .0532 & 1.960 & NS \\
\hline \multirow{11}{*}{$\begin{array}{l}\text { Fabrication/ } \\
\text { Welding }\end{array}$} & & Teachers & 49 & 2.7500 & .89685 & .18307 & & & & \\
\hline & Item 14 & HOD & 12 & 3.0000 & 1.15470 & .57735 & 59 & 3.444 & 1.960 & S \\
\hline & & Teachers & 49 & 2.8333 & .96309 & .19659 & & & & \\
\hline & Item 15 & HOD & 12 & 2.5000 & 1.29099 & .64550 & 59 & -0.330 & 1.960 & NS \\
\hline & & Teachers & 49 & 2.9167 & 1.01795 & .20779 & & & & \\
\hline & Item 16 & HOD & 12 & 2.5000 & 1.00000 & .50000 & 59 & -0.349 & 1.960 & NS \\
\hline & & Teachers & 49 & 2.8750 & .74089 & .15123 & & & & \\
\hline & Item 17 & HOD & 12 & 3.2500 & .95743 & .47871 & 59 & -0.160 & 1.960 & NS \\
\hline & & Teachers & 49 & 3.1250 & .89988 & .18369 & & & & \\
\hline & Overall & HOD & 12 & 2.9000 & .82462 & .41321 & 59 & 0.717 & 1.960 & NS \\
\hline & & Teachers & 49 & 2.9000 & .71748 & .14646 & & & & \\
\hline \multirow[t]{10}{*}{4 Forging } & Item 18 & HOD & 12 & 3.0000 & .00000 & .00000 & 59 & -3.087 & 1.960 & NS \\
\hline & & Teachers & 49 & 3.0833 & .92861 & .18955 & & & & \\
\hline & Item 19 & HOD & 12 & 3.50000 & 5.7735 & .28808 & 59 & 0.580 & 1.960 & NS \\
\hline & & Teachers & 49 & 3.0833 & .88055 & .17974 & & & & \\
\hline & Item 20 & HOD & 12 & 3.0000 & .00000 & .00000 & 59 & -.132 & 1.960 & NS \\
\hline & & Teachers & 49 & 3.1250 & .89986 & .18369 & & & & \\
\hline & Item 21 & HOD & 12 & 3.2500 & 95743 & .47871 & 59 & $0 .-415$ & 1.960 & NS \\
\hline & & Teachers & 49 & 2.8750 & 89988 & 18369 & & & & \\
\hline & Overall & HOD & 12 & 3.1875 & .31458 & .15729 & 59 & 0.285 & 1.960 & NS \\
\hline & & Teachers & 49 & 3.0417 & .70190 & .14329 & & & & \\
\hline \multirow[t]{3}{*}{5 Foundry } & Item 22 & HOD & 12 & 3.0000 & .00000 & .00000 & 59 & 0.012 & 1.960 & NS \\
\hline & & Teachers & 49 & 2.6667 & .96309 & .19659 & & & & \\
\hline & Item 23 & HOD & 12 & 3.5000 & .57735 & .28868 & 59 & 045 & 1.960 & NS \\
\hline
\end{tabular}




\begin{tabular}{|c|c|c|c|c|c|c|c|c|c|}
\hline & Teachers & 49 & 3.0833 & 71728 & 14641 & & & & \\
\hline \multirow[t]{2}{*}{ Item 24} & HOD & 12 & 3.0000 & .00000 & .17289 & 59 & -9.701 & 1.960 & $\mathrm{~N}$ \\
\hline & Teachers & 49 & 3.2500 & .84699 & .25000 & & & & \\
\hline \multirow[t]{2}{*}{ Item 25} & HOD & 12 & 3.2500 & .50000 & .25000 & 59 & 0.331 & 1.960 & $\mathrm{~N}$ \\
\hline & Teachers & 49 & 3.0417 & 1.04170 & .21264 & & & & \\
\hline \multirow[t]{2}{*}{ Item 26} & HOD & 12 & 3.7500 & .50000 & .25000 & 59 & 1.036 & 1.960 & \\
\hline & Teachers & 49 & 3.0417 & .95458 & .19485 & & & & \\
\hline \multirow[t]{3}{*}{ Item 27} & HOD & 12 & 3.5000 & 1.00000 & 0.5000 & 59 & 0.450 & 1.960 & $\mathrm{~N}$ \\
\hline & Teachers & 49 & 3.0833 & .92861 & 0 & & & & \\
\hline & & & & & $6^{1.1895}$ & & & & \\
\hline \multirow[t]{2}{*}{ Item 28} & HOD & 12 & 3.2500 & 0.50000 & 2.5000 & 59 & 0.2051 & 1.960 & $\mathrm{~N}$ \\
\hline & Teachers & 49 & 3.1250 & 0.79741 & .16277 & & & & \\
\hline \multirow[t]{2}{*}{ Overall } & HOD & 12 & 3.3214 & .29451 & .14725 & 59 & 0.537 & 1.960 & \\
\hline & Teachers & 49 & 3.0417 & .59944 & .12236 & & & & \\
\hline \multirow[t]{2}{*}{ Item 29} & HOD & 12 & 3.7500 & .95743 & .47811 & 59 & 3.383 & 1.960 & $\mathrm{~S}$ \\
\hline & Teachers & 49 & 2.8750 & 0.94694 & 19330 & & & & \\
\hline \multirow[t]{2}{*}{ Item 30} & HOD & 12 & 2.2500 & .95743 & .47871 & 59 & -0.349 & 1.960 & \\
\hline & Teachers & 49 & 2.6250 & .96965 & .19793 & & & & \\
\hline \multirow[t]{2}{*}{ Item 31} & HOD & 12 & 2.0000 & 1.5470 & .57735 & 59 & 0.848 & 1.960 & \\
\hline & Teachers & 49 & 2.4583 & 1.02062 & .20833 & & & & \\
\hline \multirow[t]{2}{*}{ Item 32} & HOD & 12 & 3.0000 & .81650 & .40825 & 59 & 0.799 & 1.960 & \\
\hline & Teachers & 49 & 2.5833 & .88055 & .17974 & & & & \\
\hline \multirow[t]{2}{*}{ Item 33} & HOD & 12 & 3.5000 & .57735 & .8868 & 59 & 0.799 & 1.960 & \\
\hline & Teachers & 49 & 2.9167 & .88055 & .1974 & & & & \\
\hline \multirow[t]{2}{*}{ Item 34} & HOD & 12 & 2.7500 & 1.25831 & .62915 & 59 & 0.188 & 1.960 & \\
\hline & Teachers & 49 & 3.0000 & 97802 & .19964 & & & & \\
\hline \multirow[t]{2}{*}{ Overall } & HOD & 12 & 2.8750 & .45896 & .22948 & 59 & 0.221 & 1.960 & \\
\hline & Teachers & 49 & 2.7431 & .74045 & .15114 & & & & \\
\hline \multirow[t]{2}{*}{ Item 35} & HOD & 12 & 2.2500 & 1.25831 & .62915 & 59 & -0.661 & 1.960 & \\
\hline & Teachers & 49 & 3.04171 & 1.2208 & .22904 & & & & \\
\hline \multirow[t]{2}{*}{ Item 36} & HOD & 12 & 1.7500 & .95743 & .62915 & 59 & -0.994 & 1.960 & \\
\hline & Teachers & 49 & 0.7500 & 79400 & .22904 & & & & \\
\hline \multirow[t]{2}{*}{ Item 37} & HOD & 12 & 2.0000 & .81650 & .40825 & 59 & 0.750 & 1.960 & \\
\hline & Teachers & 49 & 2.7083 & .95458 & .19485 & & & & \\
\hline \multirow[t]{2}{*}{ Item 38} & HOD & 12 & 2.0000 & .81650 & .40825 & 59 & -0471 & 1.960 & \\
\hline & Teachers & 49 & 2.4583 & 1.02062 & .20833 & & & & \\
\hline Overall & HOD & 12 & 2.0000 & .81650 & .40825 & 59 & & 1.960 & \\
\hline & Teachers & 49 & 2.7396 & .77136 & .15745 & & & & \\
\hline Item 39 & HOD & 12 & 2.7500 & 1.25831 & .62915 & 59 & 0.151 & 1.960 & \\
\hline & Teachers & 49 & 2.6250 & 1.09594 & 22371 & & & & \\
\hline Item 40 & HOD & 12 & 2.2500 & .95743 & .47871 & 59 & -0311 & 1.960 & \\
\hline & Teachers & 49 & 2.5833 & .82970 & .16936 & & & & \\
\hline Item 41 & HOD & 12 & 2.7500 & 1.25831 & .62915 & 59 & 0.283 & 1.960 & \\
\hline & Teachers & 49 & 2.4583 & .65801 & .13431 & & & & \\
\hline Item 42 & HOD & 12 & 2.0000 & .81650 & .40825 & 59 & -0.570 & 1.960 & \\
\hline & Teachers & 49 & 2.5417 & .83297 & .17003 & & & & \\
\hline Overall & HOD & 12 & 2.5000 & 1.10349 & .55174 & 59 & -0.178 & 1.960 & \\
\hline & Teachers & 49 & 2.7024 & .42109 & .08595 & & & & \\
\hline Item 43 & HOD & 12 & 2.2500 & .95743 & .47871 & 59 & -0432 & 1.960 & \\
\hline & Teachers & 49 & 2.7083 & .95458 & .19485 & & & & \\
\hline Item 44 & HOD & 12 & 2.7083 & 1.50000 & .75000 & 59 & -0.178 & 1.960 & \\
\hline & Teachers & 49 & 3.0000 & .72232 & .14744 & & & & \\
\hline Item 45 & HOD & 12 & 2.7500 & 1.70783 & .85391 & 59 & -0.832 & 1.960 & \\
\hline & Teachers & 49 & 3.0000 & .58977 & .12039 & & & & \\
\hline Overall & HOD & 12 & 2.5000 & 1.10349 & .55174 & 59 & -0.178 & 1.960 & \\
\hline
\end{tabular}

\section{Milling \\ Machine \\ Work}

Machine

Work

8 Shaping Machine

Work

\begin{tabular}{|c|c|c|}
\hline & Item 36 & $\begin{array}{c}\text { HOD } \\
\text { Teachers }\end{array}$ \\
\hline & Item 37 & $\begin{array}{c}\text { HOD } \\
\text { Teachers }\end{array}$ \\
\hline & Item 38 & $\begin{array}{c}\text { HOD } \\
\text { Teachers }\end{array}$ \\
\hline & Overall & HOD \\
\hline $\begin{array}{l}8 \text { Shaping } \\
\text { Machine }\end{array}$ & Item 39 & $\begin{array}{c}\text { HOD } \\
\text { Teachers }\end{array}$ \\
\hline & Item 40 & $\begin{array}{c}\text { HOD } \\
\text { Teachers }\end{array}$ \\
\hline & Item 41 & $\begin{array}{c}\text { HOD } \\
\text { Teachers }\end{array}$ \\
\hline & Item 42 & $\begin{array}{c}\text { HOD } \\
\text { Teachers }\end{array}$ \\
\hline & Overall & $\begin{array}{c}\text { HOD } \\
\text { Teachers }\end{array}$ \\
\hline 9 Design & Item 43 & $\begin{array}{c}\text { HOD } \\
\text { Teachers }\end{array}$ \\
\hline & Item 44 & $\begin{array}{c}\text { HOD } \\
\text { Teachers }\end{array}$ \\
\hline & Item 45 & $\begin{array}{c}\text { HOD } \\
\text { Teachers }\end{array}$ \\
\hline & Overall & HOD \\
\hline
\end{tabular}




\begin{tabular}{|c|c|c|c|c|c|c|c|c|c|c|}
\hline \multirow{9}{*}{$\begin{array}{c}10 \\
\text { Finishing }\end{array}$} & & Teachers & 49 & 2.7024 & .42109 & .08595 & & & & \\
\hline & \multirow[t]{2}{*}{ Item 46} & HOD & 12 & 2.5000 & 1.29099 & .64500 & 59 & 0.541 & 1.960 & NS \\
\hline & & Teachers & 49 & 3.1250 & & .15123 & & & & \\
\hline & \multirow[t]{2}{*}{ Item 47} & HOD & 12 & 2.2500 & .95743 & .47871 & 59 & 0.0671 & 1.960 & NS \\
\hline & & Teachers & 49 & 2.9583 & .75060 & .15322 & & & & \\
\hline & \multirow{2}{*}{ Item 48} & HOD & 12 & 2.0000 & .81650 & .40825 & 59 & -1.171 & 1.960 & NS \\
\hline & & Teachers & 49 & 3.0833 & .77553 & .15830 & & & & \\
\hline & \multirow[t]{2}{*}{ Overall } & HOD & 12 & 2.2500 & .95743 & .47871 & 59 & -0.799 & 1.960 & NS \\
\hline & & Teachers & 49 & 3.0556 & .68572 & .13997 & & & & \\
\hline \multirow{16}{*}{$\begin{array}{l}11 \\
\text { Soldering/ } \\
\text { Brazing }\end{array}$} & \multirow[t]{2}{*}{ Item 49} & HOD & 12 & 2.0000 & 1.41421 & 70711 & 59 & -0.981 & 1.960 & NS \\
\hline & & Teachers & 49 & 3.1250 & .79741 & 16277 & & & & \\
\hline & \multirow[t]{2}{*}{ Item 50} & HOD & 12 & 2.5000 & 1.2099 & .64550 & 59 & 1.314 & 1.960 & NS \\
\hline & & Teachers & 49 & 2.9167 & .65386 & .13341 & & & & \\
\hline & \multirow[t]{2}{*}{ Item 51} & HOD & 12 & 2.2500 & .95743 & .47871 & 59 & -0.656 & 1.960 & NS \\
\hline & & Teachers & 49 & 2.9167 & .71728 & .14641 & & & & \\
\hline & \multirow[t]{2}{*}{ Item 52} & HOD & 12 & 2.7500 & 1.25831 & .62915 & 59 & -044 & 1.960 & NS \\
\hline & & Teachers & 49 & 2.8333 & .76139 & .15542 & & & & \\
\hline & \multirow[t]{2}{*}{ Item 53} & HOD & 12 & 2.2500 & 1.25831 & .62915 & 59 & -0.750 & 1.960 & NS \\
\hline & & Teachers & 49 & 3.1250 & .85019 & .17354 & & & & \\
\hline & \multirow[t]{2}{*}{ Item 54} & HOD & 12 & 2.7500 & 1.28831 & .62915 & 59 & -0.350 & 1.960 & NS \\
\hline & & Teachers & 49 & 3.1667 & .63702 & .13003 & & & & \\
\hline & \multirow[t]{2}{*}{ Item 55} & HOD & 12 & 2.2500 & 1.25831 & .6251 & 59 & -0.683 & 1.960 & NS \\
\hline & & Teachers & 49 & 3.0417 & & .12739 & & & & \\
\hline & \multirow[t]{2}{*}{ Overall } & HOD & 12 & 2.3929 & 1.16861 & .58430 & 59 & 0.560 & 1.960 & NS \\
\hline & & Teachers & 49 & 3.0179 & .53004 & .10819 & & & & \\
\hline \multirow{12}{*}{$\begin{array}{l}12 \\
\text { Hammering } \\
\text { Metal Work }\end{array}$} & \multirow[t]{2}{*}{ Item 56} & HOD & 12 & 2.5000 & 1.00000 & .50000 & 59 & -0.764 & 1.960 & NS \\
\hline & & Teachers & 49 & 3.2917 & .62409 & .12739 & & & & \\
\hline & \multirow{2}{*}{ Item 57} & HOD & 12 & 2.5000 & 1.00000 & .50000 & 59 & -0.470 & 1.960 & NS \\
\hline & & Teachers & 49 & 3.0000 & .83406 & .17025 & & & & \\
\hline & \multirow[t]{2}{*}{ Item 58} & HOD & 12 & 2.7500 & 1.25831 & .62915 & 59 & -0.235 & 1.960 & NS \\
\hline & & Teachers & 49 & 3.0417 & .69025 & .14090 & & & & \\
\hline & Item 59 & HOD & 12 & 2.7500 & 1.25831 & .67915 & 59 & -0.312 & 1.960 & NS \\
\hline & & Teachers & 49 & 3.1250 & .61237 & .12500 & & & & \\
\hline & Item 60 & HOD & 12 & 2.5000 & 1.29099 & .64550 & 59 & 0.713 & 1.960 & NS \\
\hline & & Teachers & 49 & 3.3333 & .564466 & .11526 & & & & \\
\hline & Overall & HOD & 12 & 2.6000 & 1.11952 & .55976 & 59 & 0.502 & 1.960 & NS \\
\hline & & Teachers & 49 & 3.1583 & .44126 & .09007 & & & & \\
\hline 13 & Item 61 & HOD & 12 & 2.5000 & 1.29099 & .64550 & 59 & -0.633 & 1.960 & NS \\
\hline Measuring & & Teachers & 49 & 3.2500 & .60792 & .12240 & & 5 & & \\
\hline & Item 62 & HOD & 12 & 3.500 & .57735 & .28868 & 59 & -0.411 & 1.960 & NS \\
\hline & & Teachers & 49 & 3.2083 & .83297 & .17003 & & & & \\
\hline & Item 63 & HOD & 12 & 3.2500 & .50000 & .25000 & 59 & 0.562 & 1.960 & NS \\
\hline & & Teachers & 49 & 2.8750 & .89988 & .18369 & & & & \\
\hline & Overall & HOD & 12 & 3.0833 & .41944 & .20972 & 59 & 0.024 & 1.960 & NS \\
\hline & & Teachers & 49 & 3.1111 & .52628 & .10743 & & & & \\
\hline 14 & Item 64 & HOD & 12 & 3.0000 & .81650 & .40825 & 59 & -0.170 & 1.960 & NS \\
\hline Riveting & & Teachers & 49 & 3.1667 & .96309 & .19659 & & & & \\
\hline & Item 65 & HOD & 12 & 3.0000 & .00000 & .00000 & 59 & -2.186 & 1.960 & NS \\
\hline & & Teachers & 49 & 3.0417 & .46431 & .9478 & & & & \\
\hline & Item 66 & HOD & 12 & 3.5000 & 57735 & .28868 & 59 & 0.520 & 1.960 & NS \\
\hline & & Teachers & 49 & 3.1250 & .74089 & .15123 & & & & \\
\hline & Item 67 & HOD & 12 & 3.7560 & .95743 & .47871 & 59 & 0.846 & 1.960 & NS \\
\hline
\end{tabular}




\begin{tabular}{lcccllllll}
\multirow{3}{*}{ Overall } & Teachers & 49 & 2.9583 & 1.04170 & .21264 & & & \\
& HOD & 12 & 3.1375 & .23936 & .11968 & 59 & 0.0508 & 1.960 & NS \\
& Teachers & 49 & 3.0729 & .50798 & .10369 & & & & \\
\hline
\end{tabular}

The Mean Difference is Significant at the .05 levels.

Table 2 shows that the result of T-test and data relating to the adequacy of Mechanical Technology Curriculum content indicated there was no significant difference in the response from the two groups of respondents at 0.05level of significance for all items of the main themes in the table. Except however, there was significant difference in three of the items that is item 5, 14, and 29. Since, majority of the items (i.e. 96 percent) were upheld it then means there was no significant difference from the respondents' responses. Therefore, hypothesis $\left(\mathrm{H}_{\mathrm{O}}\right)$ was accepted for the 96 percent of the items and rejected for the remaining 4 percent of the items.

\section{DISCUSSION OF FINDINGS}

The findings have shown that the curriculum content of Mechanical Technology in 57.3 percent of the items in those themes is insignificant or rather inadequate. This will no doubt affect the students in acquiring the necessary skills that would enable them to be self-employed or hired or further their education. According to Sara (2008) noted that unless and until the curriculum content of technical college programme is comprehensive and clear, there cannot be meaningful teaching-learning process. If the training needs of the students are not met, they are bound to lose interest in the teaching learning process (Ogwo and Oranu 2006). Consequently the students when graduated would now become half-baked and there by affecting their livelihood.

An adequate Curriculum content of Technical and Vocational Education and Training Programme must, among other things be valid, Significant, Comprehensive, learnable, arrange in sequential order and must be of interest to the learner (Okoro, 1993). Thus the findings have shown that there is need for the curriculum content of Mechanical Technology Programme to be reviewed.

\section{CONCLUSION}

This study has provided empirical data to show that the mechanical technology programme in technical colleges of Kaduna and Niger states is needful and useful for training individuals for self-employment and for further education in public schools. The mechanical technology programme can be more effective if the curriculum content is adequate and taught by teachers who are professionally qualified, there is no doubt the objectives of implementing the programme can be achieved as desired by all the stakeholders.

\section{RECOMMENDATIONS}

Based on the findings of the study, the following recommendations were made:

1. Government should ensure that adequate training facilities are provided. Moreover, government should ensure existing facilities for the implementation of mechanical technology programme in technical colleges are updated.

2. Government should ensure that adequate professionally qualified teachers are employed to teach in the technical colleges. This is to ensure that the right skills, attitude and knowledge are imparted to the students

3. Government should call curriculum experts to a conduct a general review of the content of mechanical technology when the need arose. If this is done, the training needs of the students will be taken care of.

\section{REFERENCES}

[1] Bature, I.I. (2011). Evaluation of the mechanical technology programme of technical colleges in Kaduna state, Nigeria. (Unpublished Doctoral Thesis), University of Nigeria, Nsukka.

[2] Bature, I.I. (2006). Technical teacher's training programme in Nigeria for sustainable national development. Journal of Educational Research and Development. Faculty of Education Ahmadu Bello University Zaria Vol.1 No.3.

[3] Christopher, R. (1996). Curriculum adequacy and quality in high school enrolling fewer than 400 pupils $(9$-12). Eric Digest.

[4] Federal Government of Nigeria (2008). National Business and Technical Examination Board (NABTEB). Syllabus. Benin.

[5] Nzewi, U; Okpara,E.N; Akudolu, L.R.\& Anyamu, E.N. Eds (1996). Curriculum theory and planning. Nsukka Trust Publishers.

[6] Ogwo, B.A. (1996). System approach in education. In B.A. Ogwo (ed) curriculum development and educational technology. Makurid Onaire Printing \& Publishing Co. Ltd.

[7] Ogwo, B.A. \& Oranu, R.N. (2006). Methodology in formal and non-formal technical/vocational education. Enugu University of Nigeria Press Ltd.

[8] Olaitan, S.O. \& Ali, A. (1997). The making of a curriculum theory, process, product and evaluation. Onitsha cape publishers international.

[9] Okoro, O.M. (1993). Principles and methods in vocational and technical education. Nsukka: University Trust Fund.

[10] Sara, H.A. (2008). Evaluation of vocational training programme in reformatory institutions in Northern Nigeria (unpublished Doctoral Thesis). University of Nigeria, Nsukka. 